\title{
Do orientation and substrate influence apparent turning biases by the 7-spot ladybird, Coccinella septempunctata?
}

\author{
Rosalind K. Humphreys * and Graeme D. Ruxton \\ School of Biology, University of St Andrews, Dyer's Brae House, St Andrews, Fife, KY16 \\ 9TH, UK \\ *Corresponding author's e-mail address: rosalindkh08@gmail.com
}

Received 11 October 2019; initial decision 21 November 2019; revised 20 December 2019; accepted 21 December 2019; published online 24 February 2020

\begin{abstract}
How foraging predators explore their environment is a fundamental aspect of predator-prey interactions. Girling et al. (2007) tested Coccinella septempunctata in a Y-maze, finding that approximately $45 \%$ of individuals displayed significant turning biases. We extend the work of Girling et al. in three ways: (1) turning bias was tested on vertical as well as horizontal structures, (2) turning bias was tested on natural Y-shaped twigs as well as artificial twigs and (3) turning bias was recorded both as the pre-designated 'left' or 'right' physical branch selected and from the perspective of ladybirds. No significant patterns of 'handedness' were apparent with vertical orientation, on either substrate. With horizontal orientation, significant turning biases were exhibited on artificial but not natural twigs. Overall, although turning biases are theoretically efficient when searching branched structures, we propose that in natural environments ladybirds will base their foraging movements on environmental cues.
\end{abstract}

\section{Keywords}

handedness, foraging efficiency, orientation, substrate, turning bias.

\section{Introduction}

The temporal and spatial dynamics of predator-prey interactions are important to the suppression of prey by predators, and so will hold significance for the fitness of both prey and predators at population scales. An important characteristic of predators that can directly influence these dynamics is the 
utilisation of particular strategies when searching for prey. Smith (1974) defined a search strategy as "a set of basic rules of scanning and locomotion which results in the effective encountering of a specific distribution of food" and a search tactic as "an adaptive change in scanning or locomotion occurring once a predator has arrived in a specific area where prey are available". Given that an organism's evolutionary fitness depends on its ability to optimise both the quantity and quality of its diet, search strategies and search tactics should be expected to be under strong natural selection to maximise intake while minimising costs (Hassell \& Southwood, 1978), including such relevant costs as time and energy expended. The spatial structure of an environment is one factor that will influence the efficiency of search and this is particularly true for predatory insects (Hassell \& Southwood, 1978). Coccinellids have been the focus of many studies on foraging behaviour, but most of these studies utilise the larvae rather than adults (Ferran \& Dixon, 1993). Further exploration of adult foraging behaviour can be expected to shed light on how these predators maximise their fitness when searching for prey.

It is reasonable to predict that, in branched plant environments, coccinellids will be under strong selection to use the most efficient mechanism to locate prey distributed in patches across a plant (Girling et al., 2007). Research suggests that plant architecture can significantly affect the foraging efficiency of ladybirds (Carter et al., 1984; Grevstad \& Klepetka, 1992; Clark \& Messina, 1998), with highly branched plants potentially facilitating greater ease of movement for predators (Reynolds \& Cuddington, 2012). The sensory abilities of ladybirds are, at present, poorly understood (Ferran \& Dixon, 1993), but while visual (Nakamuta, 1984) and chemical volatile (Ninkovic et al., 2001; Pettersson et al., 2005; Pettersson et al., 2008) cues may play some role in prey detection at least for some species, it is thought that ladybirds can only use them over short distances (Dixon, 2000). The most efficient rules for locomotion as part of extensive search, before encountering a prey patch and switching to intensive search (Hassell \& Southwood, 1978), therefore should be selected in order to maximise an individual's chances of encountering prey patches. Here our focus is on this extensive search, that is, how do ladybirds forage on plant structures ahead of encountering any prey?

Girling et al. (2007) investigated the hypothesis that seven-spot ladybird (Coccinella septempunctata) adults exhibit turning biases in a way that improves their searching efficiency when foraging in complex branching 
environments. This hypothesis stemmed from research into ants' 'outlinetracing', a search strategy that is considered to be the most efficient on unfamiliar branched structures in terms of costs and effectiveness (Jander, 1990). Outline tracing is where a foraging individual arriving at branching points will consistently choose the left or the right option and is therefore based on an insect demonstrating an innate bias to turn in one direction over another; this is referred to as 'handedness' (Girling et al., 2007). In theory, in the absence of other cues of prey location, outline-tracing cannot be improved upon as an arboreal search strategy for exploring a complex branching environment (Jander, 1990).Thus, using a Y-maze (as is typical for experiments on foraging behaviour), Girling et al. sought to test whether ladybirds show significant turning biases. They also estimated the searching efficiencies of ladybirds with various turning biases using a simulation model. Their findings suggested that $45 \%$ of tested individuals demonstrated significant turning biases and that foraging benefits increased with the degree of bias implemented by simulated individuals (Girling et al., 2007).

Further support for the existence of individual handedness has since been reported for many invertebrates (Frasnelli, 2013; Frasnelli, 2017), including ants (Hunt et al., 2014; Endlein \& Sitti, 2018), bees (Ong et al., 2017), and giant water bugs Belostoma flumineum Say (Heteroptera: Belostomatidae) (Kight et al., 2008). Bell and Niven (2014) reported an interesting context-dependent handedness in desert locusts (Schistocerca gregaria). Locusts exhibited handedness during targeted forelimb placement but not whilst walking, reminiscent of the context-dependent handedness of some vertebrates' handedness, including humans. Such handedness may reduce the computations involved in forelimb selection for targeted movements and thus be adaptive, enhancing the efficiency of the nervous system (Levy, 1977; Bell \& Niven, 2014). Recently, O'Shea-Wheller (2019) reported further evidence for invertebrate context-dependent handedness, finding that foraging honey bees (Apis mellifera) exhibit a strong rightward turning bias, accompanied by reduced decision latency when entering open cavities. However, they show no directional preference in sequential choice-mazes, where thigmotaxis instead predominates. Honeybee workers are thought to be better equipped for sensory investigation and threat response using their right antenna and eye, so this turning bias should be expected to be adaptive in the context of exploring open cavities (O'Shea-Wheller, 2019). 
Lateralisation and 'handedness' of behaviours is also widespread in vertebrates (see Gunturkun (2012) and references therein and Maciejewska et al. (2016) for an example). Left-right asymmetries in the brains (or nervous systems) and behaviour of vertebrates and invertebrates are theorised to increase individual efficiency by: avoiding duplication of functions (Levy, 1977), enabling parallel processing of information (Rogers et al., 2004), and by allowing one 'side' of the nervous system or brain hemisphere to control actions and thus prevent the simultaneous initiation of incompatible responses (Vallortigara, 2000; Ghirlanda et al., 2009). Indeed, often animals that show lateralised behaviours, such as turning biases, seem to outperform those that do not in many circumstances (McGrew \& Marchant, 1999; Güntürkün et al., 2000; Rogers et al., 2004). Handedness, therefore, may be predicted to be useful for coccinellids - alongside other animals - both as an efficient way to forage on structures in particular contexts and as an example of lateralisation that could improve the efficiency of cognition.

However, no studies have yet attempted explore the generality of Girling et al.'s (2007) findings or begin to consider the possibility of contextdependent handedness in ladybirds. This current study sought to explore the generality of turning biases in ladybirds further by extending the methodology used by Girling et al. (2007) in several ways. Firstly, we explored whether the orientation of a Y-choice-presenting set-up would influence apparent turning bias. Girling et al. (2007) presented their Y-maze horizontally so that they could directly compare their findings with the majority of other studies (that positioned similar equipment horizontally). However, Bansch (1966) found no evidence of handedness in two-spot ladybirds (Adalia bipunctata) when searching a 50-cm-high branching model tree and suggested that geotaxis was instead the salient control of behaviour. Thus, there is reason to expect that results of horizontal choice tests may not necessarily transfer to branched structures with a vertical orientation. Ladybirds can be found foraging on a very wide variety of plants with branches orientated at a great diversity of inclinations, so investigating their foraging behaviours on structures angled differently to 180 degrees could be insightful. Secondly, we compared the potential influence of substrate on turning bias, by testing ladybirds on natural and artificially-constructed twigs. Although Girling et al. (2007) improved on previous studies by including a linear wire in their Y-tube olfactometer for ladybirds to follow along, resembling the branch of a plant, this substrate may be unfamiliar to their subjects 
in its tactile properties. The exploration of turning bias in wild ladybird populations was here extended by comparing movements on Y-shaped twigs acquired from ladybirds' natural habitat with those on artificial wire twigs constructed to similar lengths. Finally, we investigated turning directions by considering both whether the selected branches were the designated 'left' or 'right' branch of twigs and whether ladybirds' selected branches were 'left' or 'right' from the perspective of the ladybird as they approached the turning decision point. We arbitrarily designated one physical branch as left and right for each twig (as Girling et al. had pre-determined 'lefts' and 'rights' for their constructed wires) and recorded both the physical branch selected and the 'ladybird's perspective' branch selected. The second approach accounted for occasions where the ladybirds may have circled round the stem of the twig before making a turning decision. That is, two ladybirds could select the same physical branch and this could be recorded as a left choice from one's perspective and a right choice from the other's perspective - if they approached the junction from opposite sides of the main starting stem (see Figure 1). We considered the 'observer reference frame' approach to give us commonality with Girling et al., but also introduced the 'ladybird perspective' approach since this more naturally reflects the behavioural processes of the ladybirds.

\section{Material and methods}

\subsection{Study organisms}

Adult seven-spot ladybirds Coccinella septempunctata L. (Coleoptera: Coccinellidae) were collected along Lade Braes walk, St Andrews during August $2019\left(56.34^{\circ} \mathrm{N}, 2.81^{\circ} \mathrm{W}\right)$. Ladybirds were kept individually in sterilised plastic tubs (4.8 cm in height, $6.5 \mathrm{~cm}$ in diameter) with a nylon mesh lid for ventilation. Ladybirds were transferred individually using a fine-haired paintbrush so as to minimise stress and injury during handling. Each container was provided with several small twigs taken from the original habitat, a piece of damp paper towel, and raisins as a food supplement, which were replaced daily. Ladybirds could not be starved $24 \mathrm{~h}$ ahead of trials (as per Girling et al., 2007) because trials for any individual ladybird took place over several days. Although raisins were provided to help keep ladybirds alive over this time, aphids are the primary dietary staple for ladybirds and so the raisins were not expected to diminish ladybirds' foraging behaviour when tested. In any case, ladybirds were rarely seen to settle on raisins when 
a.

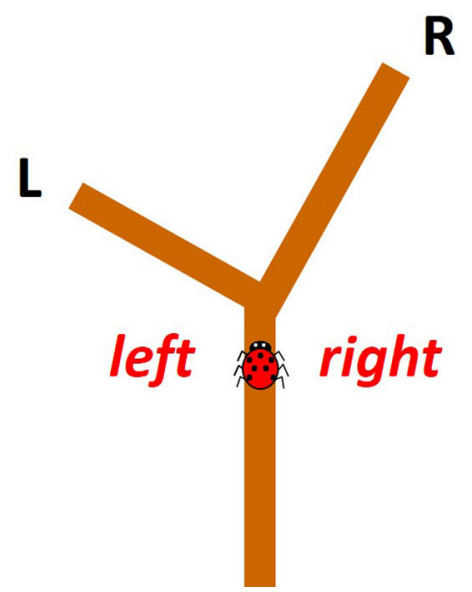

b.

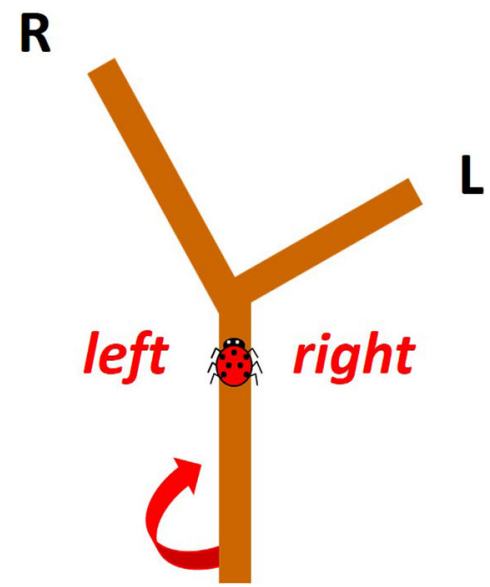

Figure 1. An example of how a ladybird's movement around the other side of a twig and subsequent approach to the decision point would affect the turning decision from the ladybird's perspective. Both images depict the same theoretical Y-shaped twig, with its longer branch designated as ' $R$ ' (right) and its shorter branch designated as 'L' (left). In situation (a) the ladybird remains on the 'front' side of the twig and so the turning choices from its perspective (italicised lower-case) match those presented by the designated branch labels ('observer reference frame' approach). In situation (b) however, the ladybird is approaching those same physical branches on the twig, but has moved around the 'back' of the twig, such that the turning choices from its perspective no longer match those presented by the designated branch labels.

in their containers and little damage was found on replaced raisins, suggesting that ladybirds will have been hungry or even starved ahead of testing and therefore highly motivated to forage. Room temperature was kept at approximately $18^{\circ} \mathrm{C}$ via a thermostat heating system and the tubs were kept by a window so that ladybirds had access to natural light cycles even when not participating in testing. No ladybird was kept for more than three days before being released.

\subsection{Design of experimental set-up}

Seven Y-shaped twigs were collected along Lade Braes walk, St Andrews $\left(56.34^{\circ} \mathrm{N}, 2.81^{\circ} \mathrm{W}\right)$. The twigs varied in height, girth, and texture, providing a range of natural variation to test the subjects on (see Figure A1 in Appendix A). Each twig was assigned a number and had the branches leading from the stem of the ' $\mathrm{Y}$ ' randomly designated as 'left' and 'right'; the left 
branch was marked with a tiny piece of gold tape. Seven artificial Y-shaped twigs were then constructed from plastic-coated garden wire, each matching to within $1 \mathrm{~cm}$ the lengths of each of the branches of one of the natural twigs (see Figure A2 in Appendix A for examples). For each trial, depending on whether a natural or artificial twig was required, a twig of that given substrate was selected randomly.

For each trial, the selected twig was affixed by blu tack in a large, sterilised clear plastic container: $20(\mathrm{H}) \times 32(\mathrm{~W}) \times 23 \mathrm{~cm}(\mathrm{D})$. In experiment 1 , the twig was orientated vertically, affixed to the centre of the base of the container such that the left-right axis of the twig was aligned with the widest length of the container (see Figure A3 in Appendix A). In experiment 2, the twig was orientated horizontally, affixed to the centre of one of the short wall sides of the container such that the left-right axis of the twig was aligned with the short wall but that the $\mathrm{Y}$ pointed into the centre of the container (see Figure A4 in Appendix A).

\subsection{Test for bias in individuals}

In total, 18 individual ladybirds were tested over the whole study. For each experiment, 9 individual ladybirds were tested across 3 days, running blocks of 5 trials per individual with natural twigs and 5 trials with artificial twigs on each day. Individuals experienced trials with both twig substrates in the morning (7:30 am-12:00 pm) and in the afternoon (1:30 pm-6 pm) such that the order of testing was not the same on every day. The order of testing for the ladybird individuals was randomised at the beginning of each 5-trial block. Upon selection, ladybirds were transferred to a different plastic container $(4(\mathrm{H}) \times 9(\mathrm{~W}) \times 9 \mathrm{~cm}(\mathrm{D}))$ using the paintbrush and were kept in this container between each of their trials. In total, each ladybird made 15 choice decisions on randomly-selected natural twigs and 15 on artificial ones. The relatively small sample size per experiment $(N=9)$ was seen as an unfortunate but necessary trade-off in order to run 30 trials with each individual, at different times of day and over several days, while still completing the overall study within the same short time period in the season to avoid developmental and seasonal effects becoming confounders.

At the beginning of a trial, an audio recording which ran a timer was started, ready for behaviours to be dictated to it as they occurred and for the timing of events around the movements made to be kept track of as the trial took place. The ladybird was then introduced to the base of the twig in the 
middle of the main stem from the 'front' of the twig (with the designated branches presented left to right) using the paintbrush. The ladybird was allowed to run up and down the paintbrush (to overcome handling stress) until it opted to move onto the base of the twig. Once on the base of the twig, a ladybird was given a maximum of two minutes to move up the main stem and move a minimum of $1 \mathrm{~cm}$ up one of the branches. Once this had occurred, the individual was considered as having made a turning decision and whether the selected branch was the designated left or right branch of the twig was recorded (the 'observer reference frame' approach). Whether the selected branch was left or right from the perspective of the ladybird as it approached the turning decision point was also recorded, in order to account for occasions where the ladybird had moved around to the other side of the twig stem before making a turning decision (the 'ladybird perspective' approach).

If ladybirds left the twig before reaching the branching point and making a decision, the trial was re-started. The twig branches never touched and were never in reach of the sides of the plastic container. During all trials, the observer remained at a distance, to prevent breath from influencing behaviour, and avoided casting a shadow over the equipment. As discussed in the introduction, Dixon (2000) suggested that ladybird sensory systems are attuned to short spatial scales, considerably shorter than the distance to the observer in our experiments. While we cannot be certain of whether the observer's presence influenced visual cues, the observer did not systematically vary their proximity to the apparatus or their movement. The observer was also, of course, far larger than potential predators of ladybirds would be whilst attacking and so was unlikely to have provoked responses more associated with avoiding predation than foraging. The room was well-lit by natural light, and after each trial the trial container was rotated by 45 degrees to control for the effects of any environmental gradients within the room. Unlike in the study by Girling et al. (2007), neither the natural nor the artificial twigs were cleaned between trials. Although we acknowledge that ladybirds may leave behind chemical residues on substrates they explore, we know of no evidence of ladybird sensitivity to any such residues. Further, the structures ladybirds encounter in the wild would likely be covered in residues of other organisms that have explored the plant. Cleaning down the natural substrates may have significantly impacted some of the natural traits we sought to explore the influence of, removing residues from the wild and potentially damaging the bark. It would then be unhelpful to the comparison aspect of 
this study to clean down the artificial substrates. The lack of cleaning procedure did not result in ladybirds either consistently following or avoiding the path of the previous ladybird on any given twig - artificial or natural.

\subsection{Analysis}

All analyses were conducted using R version 3.4.3 (R Core Team, 2017). For all comparisons of observed frequencies of left-or-right turning decisions in each experiment, chi-square tests were used. GLMs containing only turning choice as a factor also confirmed the findings of chi-square tests comparing the frequency of left-or-right turning decisions.

\section{Results}

\subsection{Experiment 1: vertical orientation}

Looking at the turning decisions of individuals on vertically-orientated twigs (Figure 2), on natural twigs one third of individuals (three out of nine) showed significant differences in chi-square tests concerning their selection of left-or-right across their 15 choices. In all of these cases the biases were only apparent from the designated branch labels rather than being left-orright biases from the ladybird's perspective as they approached the decision point. As each individual underwent multiple trials separately, a number of individuals should be expected to produce positive results even in the absence of any turning bias simply through type I errors. A total of 10000 replicate simulations of the experiment were conducted under the assumption of all individuals having no bias, in order to test the likelihood of these results in a situation of no true individual-level variation. Of these simulations, less than $1 \%$ produced three or more out of nine individuals with apparent turning biases through type I error alone, suggesting that the preferences shown in terms of the twigs' designated left or right branches were not simply down to type I errors. However, since there was no evidence of left-orright individual biases from a ladybird's perspective, the most parsimonious explanation is that some ladybirds did not choose branches randomly but rather selected according to some physical twig trait that was not equally balanced across branches designated left or right on the twigs that they encountered. That is, we can tentatively suggest that some ladybirds do not choose their path randomly but do not use left-or-right bias to make their non-random choice; rather, some unidentified physical trait (or traits) of the natural substrate influence turning choice. Such physical twig traits could in- 


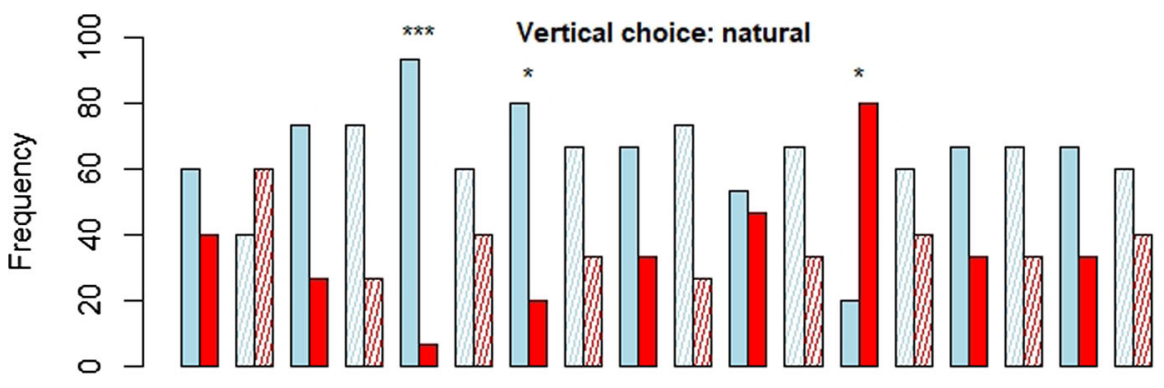
A A
B B
C C D
D $\quad$ E $\quad E \quad F \quad F$
$\begin{array}{llllllllllll} & G & G & H & H & \text { I } & \text { I }\end{array}$

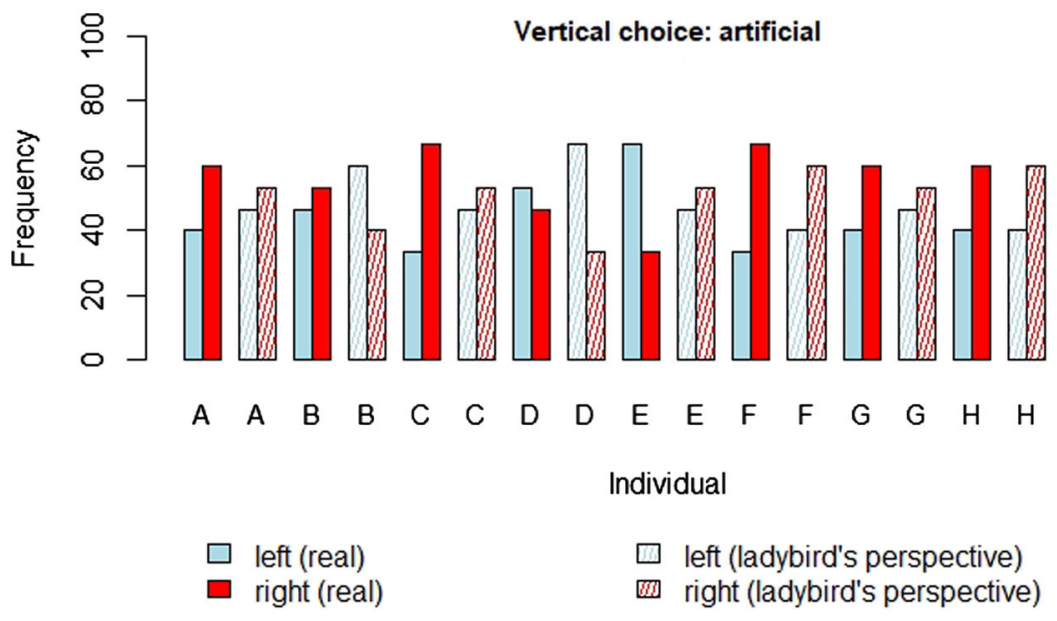

Figure 2. The $\%$ times individual ladybirds chose to turn left (blue) or right (red) on natural and artificial twigs across experiment 1 , from both the perspective of the designated branches ('real', fully-coloured bars) and the perspective of the ladybird as it reached the turning point ('ladybird's perspective', shaded bars). Asterisks indicate the chi-square test significance of the frequency of left and right decisions by different individuals, where $*<0.05$ and $* * *<$ 0.001 .

clude: the colouration, textures, patterning of the twig bark, thickness, and the structure of the branched turning choice.

Across the artificial twigs (Figure 2), only one of the nine individuals showed significant differences in chi-square tests of their left-or-right decisions, and in this case the bias was only significant as turning decisions made from the ladybird's perspective, as opposed to the designated branches. $28 \%$ of 10000 simulations assuming all ladybirds were unbiased produced at least as extreme an occurrence of turning bias though type I errors alone. From 
this, there is no strong evidence to suggest consistent bias for left or right in these ladybirds on this artificial substrate.

\subsection{Experiment 2: horizontal orientation}

Looking at the turning decisions of individuals on horizontally-orientated twigs (Figure 3), on natural horizontally-orientated twigs no individuals showed any significant differences in chi-square tests of the frequencies of left and right decisions, both based on branch designations and the la-
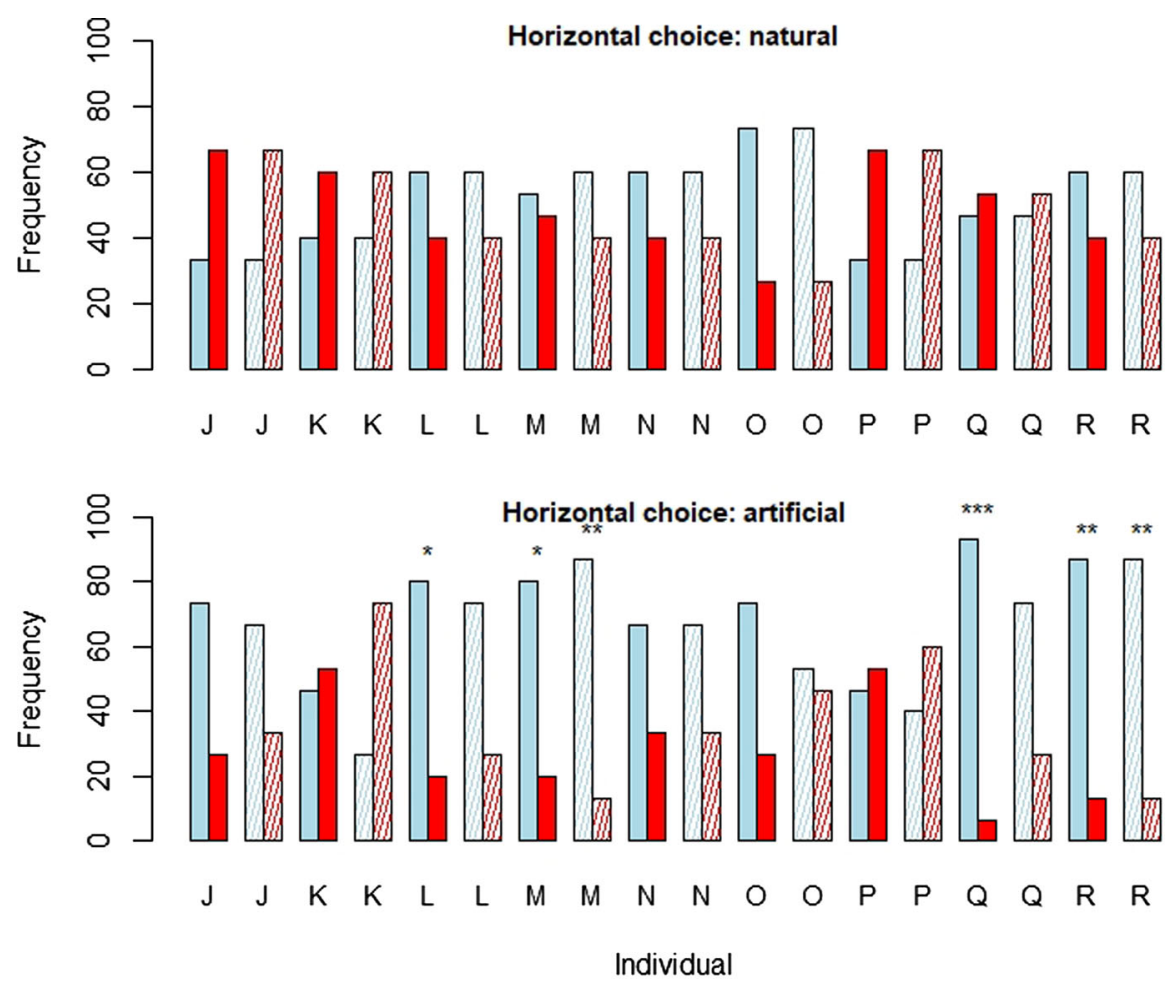

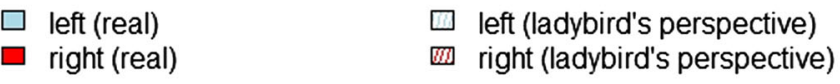

Figure 3. The $\%$ times individual ladybirds chose to turn left (blue) or right (red) on natural and artificial twigs across experiment 2 , from both the perspective of the designated branches ('real', fully-coloured bars) and the perspective of the ladybird as it reached the turning point ('ladybird's perspective', shaded bars). Asterisks indicate the chi-square test significance of the frequency of left and right decisions by different individuals, where $*<0.05$, $* *<0.01$ and $* * *<0.001$. 
dybird's perspective when approaching the turning point. On the artificial twigs, however, four of the nine individuals showed significant turning tendencies. Re-sampling simulations of 10000 runs found that less than $0.1 \%$ produced four or more out of nine individuals with turning biases through type I error alone, suggesting that the individual preferences shown in terms of the twigs' designated left or right branches were real. Individuals L, M, $\mathrm{Q}$ and $\mathrm{R}$ chose the designated left branch significantly more times than the designated right branch (Figure 3). Four of the nine individuals tested in experiment $2(44.4 \%)$, therefore, displayed significant turning biases onto the left designated branches, resembling the $45 \%$ of individuals Girling et al. (2007) found to exhibit significant turning biases (19 out of 42). However, in this current study only individuals $\mathrm{M}$ and $\mathrm{R}$ exhibited this bias to a significant degree $(p<0.01)$ from the ladybirds' perspective at the decision point (Figure 3). Re-sampling simulations found that of 10000 runs $4 \%$ produced two or more out of nine individuals with turning biases through type I error alone, suggesting that the preferences in terms of ladybirds' approach choice was likely down to individuals' bias. Two of the nine individuals tested in experiment $2(22.2 \%)$, therefore, displayed significant turning biases to the left, but this was a smaller proportion of ladybirds than the 19 out of 42 (45\%) individuals Girling et al. (2007) found to exhibit significant turning biases in their study also using a horizontally-orientated artificial substrate.

\subsection{Choices made from different perspectives}

Examining how many times ladybirds' turning choice as they approached the decision point corresponded with the pre-designated 'left' or 'right' labels of twigs (Table 1), it is clear that ladybirds moved around the other side of the branch far less frequently during trials with horizontally-orientated twigs than during trials with vertically-orientated twigs. When the set-up was vertical, ladybirds significantly more frequently made their choice from the 'front' of the twig - such that their choice matched the designated branch choice - when on natural twigs $\left(\chi_{1}^{2}=3.9185, p<0.05\right)$, but this was not the case on artificial twigs $\left(\chi_{1}^{2}=0.0074074, p=0.9314\right)$. When the set-up was horizontal, ladybirds again made more choices from the 'front' of natural twigs than on artificial twigs (Table 1), but across both substrate types far more ladybirds made their decision from the 'front' of twigs, matching the designated branch choice, than around the back (natural: $\chi_{1}^{2}=131.03$, $p<0.001$, artificial: $\left.\chi_{1}^{2}=58.674, p<0.001\right)$. Aggregating the data on matching versus differing choices, it is clear that there was a stronger ten- 
Table 1.

Frequency of trials where the left-right turning choice made from the ladybirds' perspective matched the designated 'left' or 'right' label assigned to the chosen branch for each combination of methodological set-up.

\begin{tabular}{lccr}
\hline Set-up & Matching choices & Differing choices & Total \\
\hline Vertical and natural & 79 & 56 & 135 \\
Vertical and artificial & 68 & 67 & 135 \\
Horizontal and natural & 134 & 1 & 135 \\
Horizontal and artificial & 112 & 23 & 135 \\
\hline
\end{tabular}

dency for ladybirds' turning decisions to match rather than differ with the choice of designated branch on horizontal twigs $\left(\chi_{1}^{2}=182.53, p<0.001\right)$ regardless of substrate than on vertical twigs $\left(\chi_{1}^{2}=2.1333, p=0.1441\right)$. Essentially, when on horizontally-orientated branches, ladybirds strongly preferred to walk above than below the branch. This is to be expected, since walking above the branch causes gravity to act to secure them to the branch, whereas when walking underneath they must resist the force of gravity acting to detach them. On vertically-orientated branches there is no such strong preference, likely because orientation with respect to the branch would not affect gravitational forces experienced.

\section{Discussion}

During experiment 1 (vertical orientation), in trials with the artificial twigs only one individual exhibited a significant turning bias during their trials, and this was from their perspective rather than being a bias for designated sides of twigs. However, this single incident of apparent handedness had a reasonable likelihood of resulting simply from type I error. On the natural twigs, however, three of the nine individuals in experiment 1 demonstrated significant differences in the frequencies of left and right choices they made in terms of the designated branches they selected, but none showed a significant turning bias towards either direction when considering the direction in which they turned from their approach. Together, these findings suggest that no real patterns of handedness were seen across either substrate in experiment 1 . Perhaps, as in the study by Bansch (1966), geotaxis best explains the behaviour seen with the vertical experimental set-up. That is, rather than handedness influencing movement up vertically-orientated branched structures, ladybirds may have primarily sought to move against the force 
of gravity and head upwards regardless of junctures forcing turning decisions - the turning choices themselves may be irrelevant to the ladybirds' overriding goal of moving up the structure.

Turning then to experiment 2 , the horizontal orientation of twigs resembled more the set-up of Girling et al. (2007) than experiment 1. Looking at individual turning choices across trials with natural twigs, no significant differences were seen across either the choices between designated branch labels or the turning choices from the individual's perspective for any of the ladybirds tested. It is important to note that throughout experiment 2 there was much less of a difference between the turning choice from the ladybirds' perspective and from the designated turning choices assigned to the branches than there was in experiment 1 (Table 1). With horizontally-orientated twigs, regardless of substrate, ladybirds showed a strong tendency to explore their environment while remaining on the top side of twigs as opposed to moving along below it where they would have to hang upside down. This suggests an influence of geotaxis on ladybird movement, as the difference felt in gravity will be greater in the choice between twig sides during horizontal orientation (i.e. one side involves being suspended upside down) than it is on either side of a vertically-orientated twig. In contrast to the natural twigs, when on horizontally-orientated artificial twigs in experiment 2 , four of the nine ladybirds showed significant tendencies to select the designated left branch of twigs, but only two of these individuals also exhibited a significant bias in the decision to turn left from their perspective on the approach to the decision point. Interestingly, both cases of significant bias from the ladybird's perspective involved individuals turning predominately left. While the direction of bias in cases of handedness across different animals is sometimes found to be biased itself at the population level (Hunt et al., 2014; Frasnelli \& Vallortigara, 2018; O'Shea-Wheller, 2019), Girling et al. (2007) reported that of the individuals they found to exhibit significant turning biases there were similar numbers of individuals biased to the left and right. Further consideration of population-scale bias in this current study is detailed in Appendix B. But, remaining at the individual-scale, the findings here suggest that when twigs are orientated horizontally, significant turning biases are more likely to be exhibited by individuals on artificial substrates than on natural substrates. However, anecdotally, throughout this present study it was far harder to get ladybirds to run along the horizontally-orientated twigs than the verticallyorientated twigs; far more trials had to be re-started as ladybirds would often leave the main stem before reaching the turning point to make a decision. 
This may suggest that ladybirds prefer to move along vertical branches than horizontal ones.

To conclude, when twigs were orientated vertically (experiment 1 ), little evidence was found for 'handedness' from the ladybirds' perspective on either substrate. Twigs naturally occur at a diversity of angles, but phototaxic growth may tend to encourage upward orientation. This may mean that the findings from experiment 1 are, to some extent, more likely to reflect behaviours exhibited by ladybirds in most circumstances in the wild. When twigs were orientated horizontally (experiment 2), as they were in the original study of Girling et al., more individual turning biases were seen in terms of the observer reference frame on artificial substrate than on natural substrate, but only a small proportion of individuals exhibited this 'handedness' from their perspective when approaching the turning decision. Ladybirds might be somewhat more inclined to demonstrate innate turning bias on artificial twigs that are orientated horizontally, perhaps because the lack of gravitational or light variation leaves ladybirds with fewer natural cues on which to guide foraging movements. Concerning the differences in vertical and horizontal substrate handedness, it might also be the case that ladybirds show some context-dependent handedness (Bell \& Niven, 2014; O'SheaWheller, 2019). In terms of searching for prey, it may be logical to have a bias for systematic search that involves handedness on one level of a plant structure, but the direction of turning choices may be unimportant as ladybirds move up onto higher levels. Further work on the possibility for contextdependent handedness in individual ladybirds could shed light on whether this occurs in the wild and, if so, how does it provide an adaptive function.

Predator-prey interactions are fundamentally impacted by the ways in which foraging predators explore their environment. Any search strategies or tactics employed by predators have the potential to significantly influence the fitness of prey and their predators at both individual and population scales. Theoretically, having a turning bias is a very effective search strategy for invertebrate predators in branched environments; this was demonstrated in simulations by Girling et al. (2007). However, while in the stimulus-free world of virtual coccinellids handedness may be the most efficient tool with which to locate prey patches, it is likely that in the real world there are natural cues that ladybirds could follow that are better indicators of where they could successfully find prey and, therefore, following them is preferred as a foraging strategy compared to innate turning behaviour. Perhaps ladybirds require an environment that presents the cues they might experience in the natural 
world in order to demonstrate normal foraging behaviours, such as: plant architecture, variable branch thickness, branch texture, gravity, and gradients in light and/or temperature. The recent study by O'Shea-Wheller (2019) found that foraging honey bees exhibit strong right turning bias when entering open cavities, but do not show this directional preference in sequential choice-mazes. We suggest that context may also be important in the demonstration of handedness by foraging ladybirds. Our results confirm Girling et al.'s observation (2007) that ladybirds can show handedness in their navigational decisions. However, we suggest that Girling et al.'s experimental set-up may have provided a set of circumstances (a simple artificial substrate orientated horizontally) that denies ladybirds other cues as to likely prey locations and allows handedness to be exhibited. When we deviated from this situation (by using vertical orientation and/or natural substrate) then the ladybirds may have used cues - such as gravity, or natural twig textures and architecture - more familiar to them when foraging naturally to make turning decisions, and did not appear to display innate handedness.

It is also possible that ladybirds have not been strongly selected to search plants efficiently for prey, either by using turning biases or by the most effective use of natural cues. Ladybirds may be less time-constrained than optimal foraging theory implicitly assumes, perhaps in part due to them being highly chemically-defended (and thus at low risk of predation while foraging). Thus, it may be that we should not expect ladybirds to demonstrate 'optimal' behaviour in terms of efficiency when allowed to forage naturally. In fact, coccinellid larvae usually search randomly rather than systematically for prey, with the edges and veins of leaves often determining the pattern of movement and chance playing a large part in their encountering of aphids (Banks, 1957). Larvae have also been reported as wasting time and energy repeatedly visiting parts of plants which they have already visited (Banks, 1957). Certainly, ladybirds appear to search more thoroughly once prey have been encountered, switching to intensive area-restricted search (Banks, 1957; Nakamuta, 1985; Ferran \& Dixon, 1993), but the more extensive search may not follow as efficient rules as theory predicts. Further work on the foraging behaviour of adult ladybirds would do well to track foraging for aphids on real branched plants while monitoring differences in capture rates (Girling et al., 2007) alongside variability in both traits of the plants and broader environmental factors. It may also be valuable to explore whether the behaviours seen on artificial and/or horizontal set-ups are more akin to escape behaviour than foraging behaviour. We suspect that if lady- 
birds are stressed and seeking to escape their situation they are unlikely to behave in the same manner as they would when foraging for prey in an environment which feels natural to them. Perhaps whether escape or foraging behaviour is elicited more on different set-ups could be teased apart by placing aphids on branched set-ups and observing whether ladybirds target them as prey or ignore them. However, our results presented here provide tentative evidence that handedness may not be as important a factor in ladybird search strategies than previous work has suggested. We confirm that ladybirds can indeed show handedness, but may be primarily inclined to do so when other stimuli are not available.

\section{Acknowledgements}

We thank both the Perry Foundation and the University of St Andrews for funding.

\section{References}

Banks, C.J. (1957). The behaviour of individual coccinellid larvae on plants. - Br. J. Anim. Behav. 5: 12-24.

Bansch, R. (1966). On prey-seeking behaviour of aphidophagous insects. - Academia, Prague \& Dr. W. Junk, The Hague.

Bell, A.T.A. \& Niven, J.E. (2014). Individual-level, context-dependent handedness in the desert locust. - Curr. Biol. 24: R382-R383.

Carter, M.C., Sutherland, D. \& Dixon, A.F.G. (1984). Plant structure and the searching efficiency of coccinellid larvae. - Oecologia 63: 394-397.

Clark, T.L. \& Messina, F.J. (1998). Plant architecture and the foraging success of ladybird beetles attacking the Russian wheat aphid. — Entomol. Exp. Appl. 86: 153-161.

Dixon, A.F.G. (2000). Insect predator-prey dynamics: ladybird beetles and biological control. - Cambridge University Press, Cambridge.

Endlein, T. \& Sitti, M. (2018). Innate turning preference of leaf-cutting ants in the absence of external orientation cues. - J. Exp. Biol. 221.

Ferran, A. \& Dixon, A.F.G. (1993). Foraging behaviour of ladybird larvae (Coleoptera, Coccinellidae). - Eur. J. Entomol. 90: 383-402.

Frasnelli, E. (2013). Brain and behavioral lateralization in invertebrates. - Front. Psychol. 4: 939.

Frasnelli, E. (2017). Lateralization in invertebrates. — In: Lateralized brain functions: methods in human and non-human species (Rogers, L.J. \& Vallortigara, G., eds). Springer, New York, NY, p. 153-208.

Frasnelli, E. \& Vallortigara, G. (2018). Individual-level and population-level lateralization: two sides of the same coin. - Symmetry-Basel 10: 10 . 
Ghirlanda, S., Frasnelli, E. \& Vallortigara, G. (2009). Intraspecific competition and coordination in the evolution of lateralization. - Phil. Trans. Roy. Soc. B: Biol. Sci. 364: 861-866.

Girling, R.D., Hassall, M. \& Turner, J.G. (2007). Do turning biases by the 7-spot ladybird, Coccinella septempunctata, increase their foraging efficiency? - Behaviour 144: 143163.

Grevstad, F.S. \& Klepetka, B.W. (1992). The influence of plant architecture on the foraging efficiencies of a suite of ladybird beetles feeding on aphids. - Oecologia 92: 399-404.

Güntürkün, O. (2012). Brain asymmetry in vertebrates. - In: How animals see the world: comparative behavior, biology, and evolution of vision (Lazareva, O.F., Shimizu, T. \& Wasserman, E.A., eds). Oxford University Press, Oxford, p. 501-519.

Güntürkün, O., Diekamp, B., Manns, M., Nottelmann, F., Prior, H., Schwarz, A. \& Skiba, M. (2000). Asymmetry pays: visual lateralization improves discrimination success in pigeons. - Curr. Biol. 10: 1079-1081.

Hassell, M.P. \& Southwood, T.R.E. (1978). Foraging strategies of insects. — Annu. Rev. Ecol. Systemat. 9: 75-98.

Hunt, E.R., O’Shea-Wheller, T., Albery, G.F., Bridger, T.H., Gumn, M. \& Franks, N.R. (2014). Ants show a leftward turning bias when exploring unknown nest sites. - Biol. Lett. 10: 4.

Jander, R. (1990). Arboreal search in ants: search on branches (Hymenoptera: Formicidae). J. Insect Behav. 3: 515-527.

Kight, S.L., Steelman, L., Coffey, G., Lucente, J. \& Castillo, M. (2008). Evidence of population-level lateralized behaviour in giant water bugs, Belostoma flumineum Say (Heteroptera: Belostomatidae): T-maze turning is left biased. — Behav. Proc. 79: 66-69.

Levy, J. (1977). The mammalian brain and the adaptive advantage of cerebral asymmetry. Ann. NY Acad. Sci. 299: 264-272.

Maciejewska, M., Zięba, K., Szymańska, J. \& Warońska, M. (2016). Spontaneous laterality in mouse Crl:CD1. - Laterality 21: 76-84.

McGrew, W.C. \& Marchant, L.F. (1999). Laterality of hand use pays off in foraging success for wild chimpanzees. - Primates 40: 509-513.

Nakamuta, K. (1984). Visual orientation of a ladybeetle, Coccinella septempunctata L., (Coleoptera: Coccinellidae), toward its prey. - Appl. Entomol. Zool. 19: 82-86.

Nakamuta, K. (1985). Mechanism of the switchover from extensive to area-concentrated search behaviour of the ladybird beetle, Coccinella septempunctata bruckii. - J. Insect Physiol. 31: 849-856.

Ninkovic, V., Al Abassi, S. \& Pettersson, J. (2001). The influence of aphid-induced plant volatiles on ladybird beetle searching behavior. — Biol. Contr. 21: 191-195.

Ong, M., Bulmer, M., Groening, J. \& Srinivasan, M.V. (2017). Obstacle traversal and route choice in flying honeybees: evidence for individual handedness. — PLoS ONE 12: e0184343.

O'Shea-Wheller, T.A. (2019). Honeybees show a context-dependent rightward bias. — Biol. Lett. 15: 20180877.

Pettersson, J., Ninkovic, V., Glinwood, R., Al Abassi, S., Birkett, M., Pickett, J. \& Wadhams, L. (2008). Chemical stimuli supporting foraging behaviour of Coccinella septempunctata 
L. (Coleoptera: Coccinellidae): volatiles and allelobiosis. - Appl. Entomol. Zool. 43: 315-321.

Pettersson, J., Ninkovic, V., Glinwood, R., Birkett, M.A. \& Pickett, J.A. (2005). Foraging in a complex environment - semiochemicals support searching behaviour of the seven spot ladybird. — Eur. J. Entomol. 102: 365-370.

R Core Team. (2017). R: A language and environment for statistical computing. - In: R Foundation for Statistical Computing, Vienna.

Reynolds, P.G. \& Cuddington, K. (2012). Effects of plant gross morphology on predator consumption rates. - Environ. Entomol. 41: 508-515.

Rogers, L.J., Zucca, P. \& Vallortigara, G. (2004). Advantages of having a lateralized brain. Proc. Roy. Soc. Lond. B: Biol. Sci. 271 (Suppl. 6): S420-S422.

Smith, J.N.M. (1974). The food searching behaviour of two European thrushes. II: the adaptiveness of the search patterns. - Behaviour 49: 1-60.

Vallortigara, G. (2000). Comparative neuropsychology of the dual brain: a stroll through animals' left and right perceptual worlds. - Brain Lang. 73: 189-219.

\section{Appendix A: Photos of experimental set-up}

a.

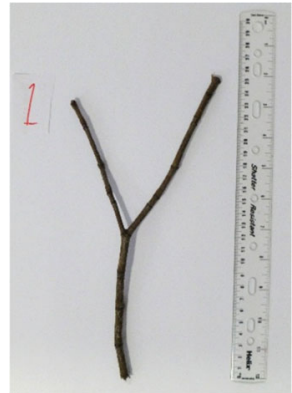

d.

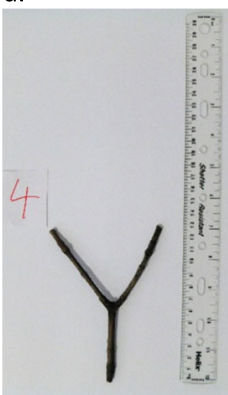

e. b.
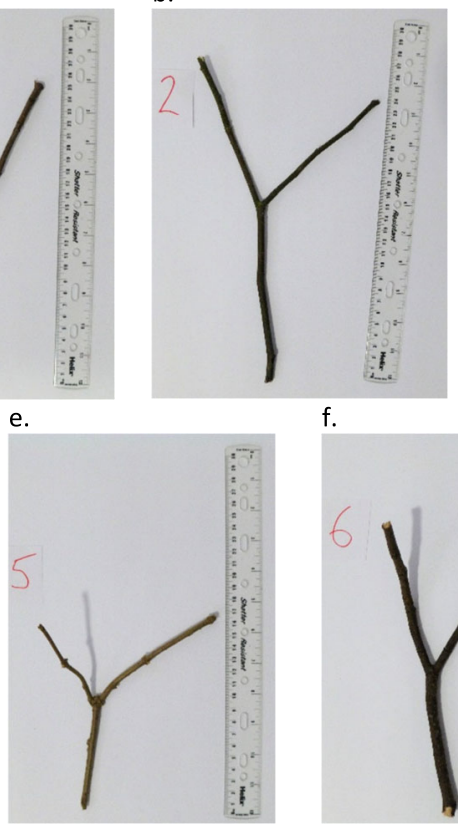

c.

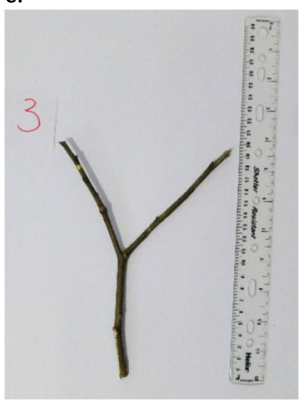

g. f.

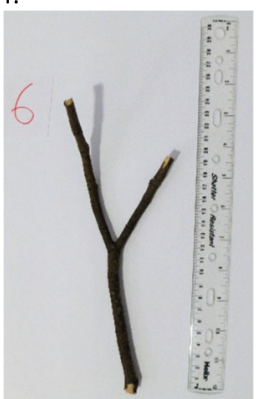

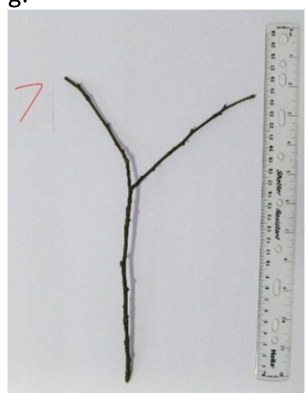

Figure A1. The 7 natural Y-shaped twigs used in both experiments 1 and 2, each alongside a $30 \mathrm{~cm}$ ruler (a-g corresponding with the twig numbers $1-7$ ). 
a.

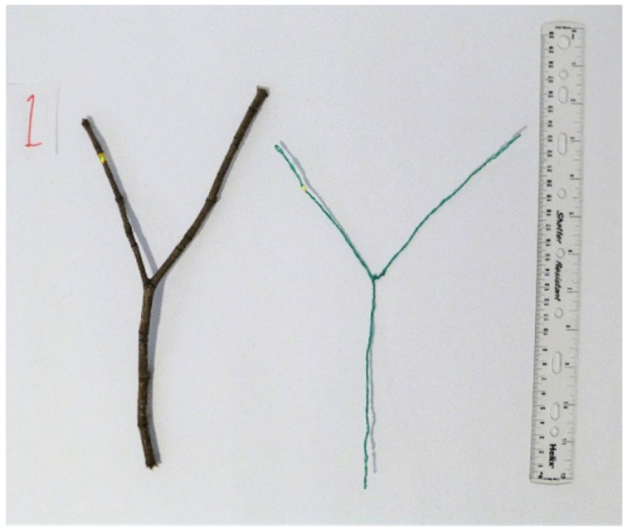

b.

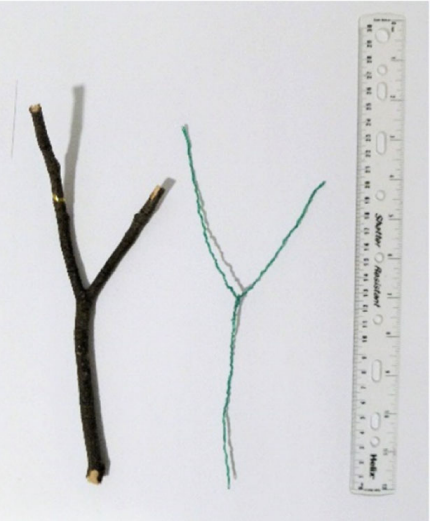

Figure A2. (a) The corresponding natural and artificial ' 1 ' twigs side-by-side alongside a $30-\mathrm{cm}$ ruler. (b) The corresponding natural and artificial ' 6 ' twigs side-by-side alongside a $30 \mathrm{~cm}$ ruler.

a.

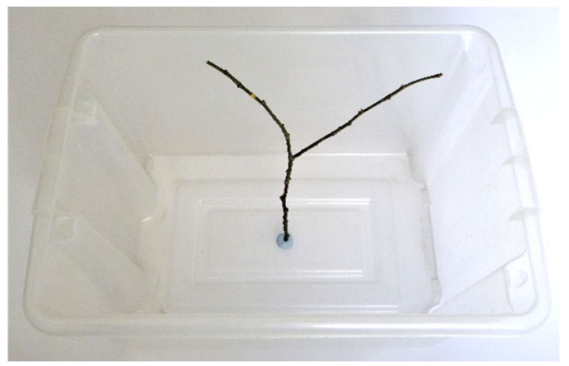

b.

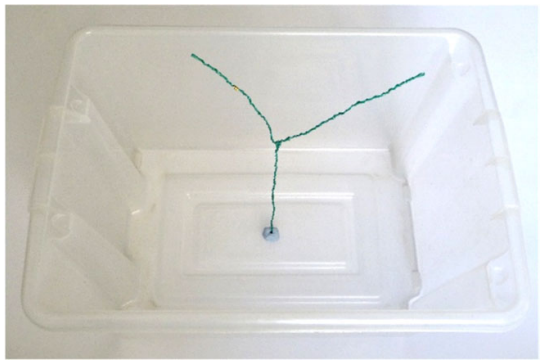

Figure A3. (a) Natural twig ' 7 ' oriented vertically for a trial. (b) Artificial twig ' 7 ' oriented vertically for a trial. 
a.

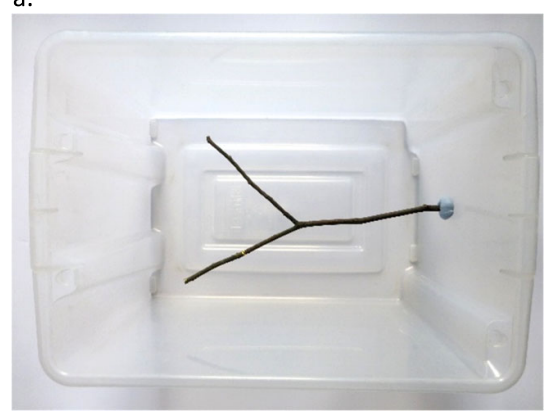

b.

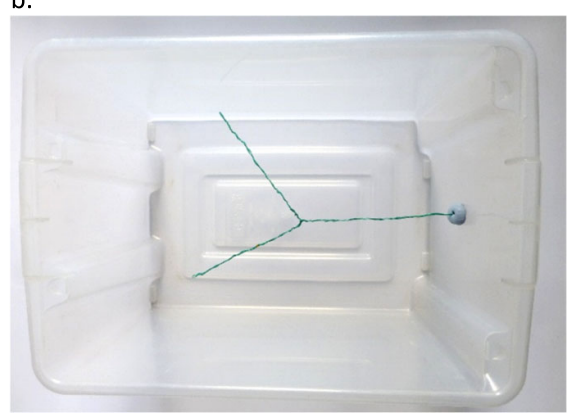

Figure A4. (a) Directly overhead view of natural twig ' 2 ' oriented horizontally for a trial. (b) Directly overhead view of artificial twig ' 2 ' oriented horizontally for a trial.

\section{Appendix B: Exploring population-level bias}

\section{B.1. Background}

Left-right asymmetries can occur at the individual- or population-level. Concerning 'handedness' in particular, individual-level asymmetry can involve an individual demonstrating significant turning bias in one direction over another, while a population-level asymmetry would involve the majority of individuals in a population exhibiting a turning bias in a particular direction over the other (Frasnelli \& Vallortigara, 2018). Behavioural and brain lateralisation at the population level is commonly found across many vertebrate species, such that within a species left- and right-type individuals occur in proportions different from one-half (Ghirlanda \& Vallortigara, 2004; Vallortigara \& Rogers, 2005; Gunturkun, 2012). While lateralisation of functions is theorised to enhance cognitive efficiency and capacity at the individual level, this adaptive advantage does not explain the alignment of the direction of behavioural asymmetries found at the population level. Rather, such biases have been proposed to arise as "evolutionarily stable strategies" under pressures occurring when individual asymmetrical organisms must coordinate their behaviour with other asymmetrical co- or conspecifics (Ghirlanda \& Vallortigara, 2004; Vallortigara \& Rogers, 2005; Ghirlanda et al., 2009).

Left-right perceptual and behavioural asymmetries — both at individual and population scales - have also been recorded in a range of invertebrates (Frasnelli, 2013, 2017; Niven \& Frasnelli, 2018). As with vertebrates, lateralisation at the population-level is suggested to have evolved as an evolutionary stable strategy in which individually asymmetrical organisms must 
coordinate their behaviour with that of other asymmetrical organisms (Frasnelli, 2013). Importantly, though, population-level asymmetries should not only be expected to be present in social species. In fact, engagement in inter-individual interactions can alone promote population-level lateralisation, as it has also been reported in the aggressive and mating displays of so-called "solitary" insects (Frasnelli \& Vallortigara, 2018). In some invertebrates, population-level turning biases have been found to relate to the species physiology. For example, foraging honey bees (Apis mellifera) exhibit a strong rightward turning bias when entering open cavities, consistent with current evidence suggesting that workers are better equipped for sensory investigation and threat response using their right antenna and eye (O'SheaWheller, 2019). The common American cockroach (Periplaneta americana) also shows an innate bias for turning right, consistent with the hypothesis that this species is right-side dominated in their tactile and odour senses (Cooper et al., 2011). Additionally, in other species such as Temnothorax albipennis ants, turning bias at a population level may serve to reduce individual predation risk through aggregation effects (Hunt et al., 2014). However, not all invertebrates that show individual-level lateralisation of behaviour exhibit population-wide lateralisation (e.g., Bell and Niven, 2014).

In the study by Girling et al. (2007), 19 of 42 individual ladybirds (Coccinella septempunctata) displayed significant turning biases when walking along a branched linear wire in a Y-maze was tested. However, a similar number of these individuals with turning biases were biased to the left and right, indicating that there was no population-level 'handedness' at play. As well as exploring individual-level biases, the present study also interpreted the findings resulting from the methodology outlined in the main text from a population level. The population-level findings are reported here.

\section{B.2. Analysis}

All analyses were conducted using R version 3.4.3 (R Core Team, 2017). For all comparisons of observed frequencies of left-or-right turning decisions in each experiment, 95\% confidence intervals (CIs) for population proportions were calculated through the normal-approximation formula for the confidence interval for a binomial proportion. 


\section{B.3. Results}

\section{B.3.1. Experiment 1: vertical orientation}

Across experiment 1, aggregating across the sample, ladybirds on verticallyorientated natural twigs chose what was the designated left branch in $64.4 \pm$ $8.1 \%$ of trials (95\% confidence intervals). That is, there was a significant population-level preference for the physically-designated left branch, found in both a chi-square test $\left(\chi_{1}^{2}=11.267, p<0.001\right)$ and in a GLM containing just the designated left or right turning choice $(p<0.001)$. Ladybirds chose what was the left branch from their perspective approaching the turning point in $63.0 \pm 8.1 \%$ of trials (95\% confidence intervals). This was also confirmed to be significant by both a chi-square test $\left(\chi_{1}^{2}=9.0741, p<0.01\right)$ and a GLM containing just the ladybirds' left or right turning choice ( $p<$ 0.01 ). From both perspectives, then, there appeared to be a slight populationlevel average tendency towards turning left on the vertically-oriented natural twigs.

Across the artificial twigs of experiment 1, there did not appear to be any overall population-level average propensity to turn one way over the other. Ladybirds chose what was designated as the designated left branch in $43.7 \pm 8.4 \%$ of trials ( $95 \%$ confidence intervals), with no significant difference in left-or-right frequencies found in either a chi-square test $\left(\chi_{1}^{2}=\right.$ 2.1407, $p=0.1434$ ) or in a GLM containing just the designated left or right turning choice $(p=0.144)$. Ladybirds chose what was the left branch from their perspective approaching the turning point in $45.9 \pm 8.4 \%$ of trials (95\% confidence intervals) and, again, no significance was found between these frequencies by either a chi-square test $\left(\chi_{1}^{2}=0.8963, p=0.3438\right)$ or in a GLM containing just the ladybirds' left or right turning choice ( $p=$ 0.344). Thus, in contrast to natural twigs, there appeared to be no evidence of a population-level average tendency towards turning left on the verticallyoriented artificial twigs.

\section{B.3.2. Experiment 2: horizontal orientation}

Across experiment 2, on the horizontally-orientated natural twigs there did not appear to be any overall population-level propensity to turn one way over the other. Ladybirds chose the designated left branch in $51.1 \pm 8.4 \%$ of trials (95\% confidence intervals), with no significant difference in left-orright frequencies found in either a chi-square test $\left(\chi_{1}^{2}=0.067, p=0.7963\right)$ or in a GLM containing just the designated left or right turning choice ( $p=$ 
0.796). Ladybirds chose what was the left branch from their perspective approaching the turning point in $51.9 \pm 8.4 \%$ of trials $(95 \%$ confidence intervals), which was also found to have no significance in either a chi-square test $\left(\chi_{1}^{2}=0.18519, p=0.667\right)$ or a GLM containing just the ladybirds' left or right turning choice $(p<0.01)$.

Across the artificial twigs of experiment 2, ladybirds chose the designated left branch in $71.9 \pm 7.6 \%$ of trials (95\% confidence intervals). There was a significant difference in the left and right frequencies from the designated branches' perspective, found in both a chi-square test $\left(\chi_{1}^{2}=25.785, p<\right.$ 0.001 ) and in a GLM containing just the designated left or right turning choice $(p<0.001)$. Ladybirds chose what was the left branch from their perspective approaching the turning point in $63.7 \pm 8.1 \%$ of trials $(95 \%$ confidence intervals). This was also confirmed to be significant by both a chi-square test $\left(\chi_{1}^{2}=10.141, p<0.01\right)$ and a GLM containing just the ladybirds' left or right turning choice $(p<0.01)$. From both perspectives, then, there appeared to be a slight population tendency towards turning left on the horizontally-orientated artificial twigs.

\section{B.4. Conclusions}

During experiment 1 (vertical orientation), in trials with the artificial twigs there did not appear to be an overall sample-wide turning bias towards any one direction in the population, either from the perspective of how the branches were designated or from the perspective of ladybirds as they approached the turning point. On the natural twigs, however, there seemed to be an overall preference of ladybirds to make a turning choice to the left, both from their perspective ahead of the choice and from the perspective of the designated branches. Turning to experiment 2, across the trials using natural twigs, ladybirds showed no overall preference to turn left or right, either in terms of the designated branch labels or in terms of their approach to the turning point. However, conversely to experiment 1 , it was during trials with artificial twigs in experiment 2 that ladybirds showed an overall propensity to turn both onto the designated left branch of twigs and to what was their left as they approached the decision point.

These rather mixed results suggest to us that further exploration of the possibility of population-level turning biases in ladybirds across a variety of situations could be valuable, both in developing understanding of their foraging behaviour and their sensory abilities. Perhaps, as with some other 
invertebrates, ladybird physiology might be such that we should predict them to exhibit population-level turning bias to the left (Cooper et al., 2011; O'Shea-Wheller, 2019). In social insects, population-level lateralisation of behaviours can be important for coordination (see Niven and Frasnelli (2018) and references therein), but this would be expected to be of lesser importance to largely-solitary ladybirds, and could potentially even reduce foraging success for an individual if it tended to search for prey in areas already explored by others. Girling et al. (2007) found no evidence of population-level turning bias in ladybirds, with a similar number of individuals demonstrating significant turning bias to left and right. Future work on ladybird foraging may help shine some light on whether ladybirds do in fact demonstrate populationlevel turning biases in different contexts and, if so, under what conditions might they have evolved.

\section{References}

Bell, A.T.A. \& Niven, J.E. (2014). Individual-level, context-dependent handedness in the desert locust. - Curr. Biol. 24: R382-R383.

Cooper, R., Nudo, N., Gonzalez, J.M., Vinson, S.B. \& Liang, H. (2011). Side-dominance of Periplaneta americana persists through antenna amputation. - J. Insect Behav. 24: 175-185.

Frasnelli, E. (2013). Brain and behavioral lateralization in invertebrates. - Front. Psychol. 4: 939.

Frasnelli, E. (2017). Lateralization in invertebrates. — In: Lateralized brain functions: methods in human and non-human species (Rogers, L.J. \& Vallortigara, G., eds). Springer, New York, NY, p. 153-208.

Frasnelli, E. \& Vallortigara, G. (2018). Individual-level and population-level lateralization: two sides of the same coin. - Symmetry-Basel 10: 10.

Ghirlanda, S. \& Vallortigara, G. (2004). The evolution of brain lateralization: a gametheoretical analysis of population structure. - Proc. Roy. Soc. Lond. B: Biol. Sci. 271: 853-857.

Ghirlanda, S., Frasnelli, E. \& Vallortigara, G. (2009). Intraspecific competition and coordination in the evolution of lateralization. — Phil. Trans. Roy. Soc. B: Biol. Sci. 364: 861-866.

Girling, R.D., Hassall, M. \& Turner, J.G. (2007). Do turning biases by the 7-spot ladybird, Coccinella septempunctata, increase their foraging efficiency? - Behaviour 144: 143163.

Güntürkün, O. (2012). Brain asymmetry in vertebrates. - In: How animals see the world: comparative behavior, biology, and evolution of vision (Lazareva, O.F., Shimizu, T. \& Wasserman, E.A., eds). Oxford University Press, Oxford, p. 501-519. 
Hunt, E.R., O'Shea-Wheller, T., Albery, G.F., Bridger, T.H., Gumn, M. \& Franks, N.R. (2014). Ants show a leftward turning bias when exploring unknown nest sites. - Biol. Lett. 10: 4.

Niven, J.E. \& Frasnelli, E. (2018). Chapter 1 - Insights into the evolution of lateralization from the insects. - In: Progress in brain research (Forrester, G.S., Hopkins, W.D., Hudry, K. \& Lindell, A., eds). Elsevier, Amsterdam, p. 3-31.

O'Shea-Wheller, T.A. (2019). Honeybees show a context-dependent rightward bias. — Biol. Lett. 15: 20180877.

Vallortigara, G. \& Rogers, L.J. (2005). Survival with an asymmetrical brain: advantages and disadvantages of cerebral lateralization. - Behav. Brain Sci. 28: 575-589. 